\title{
Ce que la descente du hamac dit du genre chez les Wayãpi de Camopi
}

\author{
OLIVIA BRACONNIER \\ Université Toulouse Jean Jaurès - Laboratoire LEEISA | Cayenne, Guyane Française \\ olbraconnier@gmail.com \\ SILVINE WALACOU \\ Secrétaire au centre de santé de Camopi | Camopi, Guyane Française \\ walacousylvine@yahoo.fr
}

DOI 10.11606/issn.2316-9133.v30i2pe193360

synthèse Notre article concerne l'expérience des femmes wayãpi de la commune de Camopi, en Guyane française, et porte spécifiquement sur les prescriptions comportementales et les traitements qui encadrent le moment des premières règles. Le corps des jeunes filles est alors considéré comme particulièrement sensible aux agressions des entités malveillantes. Le rituel de la descente du hamac organisé à la fin de la réclusion des premières règles constitue par ailleurs un moment de reproduction parallèle, car les femmes des générations précédentes l'organisent afin de transmettre aux jeunes filles des capacités physiques et mentales telles que la motivation au travail et la résistance à la douleur. Cependant, nos interlocutrices wayãpi mettent en avant l'équivalence entre le rituel des premières règles pour les femmes et celui de la naissance du premier enfant pour les hommes, montrant ainsi que les principales capacités attendues des adultes sont les mêmes, quel que soit leur genre.

Mots-clés genre, Amazonie, rituel, puberté, femmes

\begin{abstract}
What the descent of the hammock says about gender among the Wayãpi of Camopi

abstract The article concerns the experience of Wayãpi women in the commune of Camopi, in French Guiana, and deals specifically with the behavioral prescriptions and treatments that frame the moment of their first period. The bodies of young girls are then considered to be particularly sensitive to attacks from malicious entities. The ritual of descending from the hammock organized at the end of the confinement of the first period constitutes a parallel moment of reproduction, because the women of previous generations organize it in order to transmit to young girls physical and mental capacities such as motivation. at work and resistance to pain. However, our Wayãpi interlocutors highlight the equivalence between the ritual of the first menstruation for women and that of the birth of the first child for men, thus showing that the main capacities expected of adults are the same, whatever their gender. .
\end{abstract}

keywords gender, Amazon, ritual, puberty, women 


\section{Introduction}

Notre étude se déroule à Camopi, en Guyane française, chez les Wayãpi, un peuple de langue tupi-guarani sédentarisé sur ce territoire au cours du XIXème siècle. ${ }^{1}$ Nous tenterons ici de saisir les ressorts d'un moment particulier du devenir-femme dans cette société: le rituel des premières règles.

Cet article s'appuie sur des entretiens menés auprès de vingt femmes wayãpi entre janvier et mars 2021 . Nous avons des approches très complémentaires à propos du contenu de ces témoignages. L'une de nous deux est en effet une française métropolitaine qui, avant de s'orienter vers la recherche, occupait un poste d'enseignante à l'école de Camopi, tandis que l'autre est une habitante wayãpi qui connaît de façon plus intime les femmes mentionnées et a expérimenté dans son propre corps les gestes rituels relatés plus bas. Le dialogue qui s'est établi entre nous à propos de l'expérience des femmes wayãpi nous a ainsi permis d'exprimer certaines idées rapportées ici.

Nous chercherons d'abord à définir ce que produit le rituel sur les différentes actrices et sur leurs relations, tout en nous interrogeant sur ce qu'il révèle des attentes de la société vis-à-vis des femmes.

Après avoir présenté les différentes étapes du rituel, nous essayerons de déterminer en quoi celui-ci constitue un moment-clé de la fabrication des femmes par les femmes. Nous nous pencherons également sur le pendant masculin de cette étape, ce qui permettra de questionner l'importance du genre dans la construction des personnes adultes par la société wayãpi.

\section{Le rituel des premières règles}

$\mathrm{Au}$ moment de ses premières règles, une jeune fille wayãpi est d'abord recluse pendant environ une semaine dans son hamac, puis différents traitements lui sont appliqués au cours d'une nuit et de la journée suivante. Elle reste ensuite isolée jusqu'à ses deuxièmes règles, au moment desquelles un second rituel a parfois lieu. Elle continue à suivre un certain nombre de prescriptions, particulièrement alimentaires, jusqu'à ce que ses cheveux aient repoussé. Ce protocole est très courant dans les basses terres d'Amérique du Sud. Il a par exemple été documenté à propos des Ashaninka (Rojas, 1992), des Yanesha (Santos Granero, 1991), des Mai Huna (Bellier, 1991), des Shipibo-Conibo (Morin et Saladin d'Anglure, 2007), des Airo Pai (Belaunde, 2001), ou encore des Toba (Tola, 2009).

Nous nous pencherons ici principalement sur la cérémonie qui suit la réclusion initiale.

La première difficulté qui se présente pour décrire cette cérémonie est sa dénomination. Lorsqu'ils en parlent à un étranger, les Wayãpi emploient parfois le terme maraké, un mot dont l'origine est attribuée aux Wayana et qui prête à confusion, car il peut ne désigner que la phase de piqûre par les fourmis ou bien, sous la forme d'une synecdoque, toute la séquence rituelle. Dans les énoncés en langue wayãpi que j’ai récoltés, aucun

\footnotetext{
${ }^{1}$ Cette étude a été menée dans le cadre d'un master 2 d'anthropologie soutenu à l'Université de Toulouse Jean Jaurès (Braconnier 2021), et financé et encadré par le laboratoire LEEISA (CNRS - Université de Guyane Ifremer) à Cayenne.
} 
substantif spécifique n'est employé pour parler de ce qui suit la descente du hamac. Nous avons cependant noté des équivalences comme celle formulée par Barbara ${ }^{2}$, qui répondait à une de nos questions sur une action mise en place en prévision de ce moment:

C'est pour faire le maraké pour elle! O-mo-wiyt ta lem!.

Le verbe wiyi signifie descendre, et le morphème de dérivation mo est la marque du factitif. L'énoncé o-mo-wiyi lemẽ pourrait donc être traduit par : « quand on [la] fera descendre [du hamac] ». Ce rituel sera donc dénommé ici « la descente du hamac». Afin d'en offrir une description extensive, nous proposons ci-dessous le récit de la descente du hamac de Marina, jeune sœur d'une de mes principales interlocutrices.

\section{La descente du hamac de Marina ${ }^{3}$}

Marina est recluse dans son hamac attaché en hauteur aux poteaux de la maison familiale depuis que ses premières règles se sont déclenchées, il y a une semaine. Cette nuit-là, sa mère et ses grands-mères ont décrété qu'était arrivé le moment de la faire descendre.

À trois heures du matin, les membres de la famille proche commencent à se réunir autour du feu allumé sous le carbet ${ }^{4}$ de fête. Quelques rires des sœurs, tantes et cousines s'entremêlent et résonnent dans le silence de la nuit, tandis que circule une calebasse de bière de manioc (appelé localement cachiri) préparée par la mère de Marina et sa grande sœur pendant son isolement. Sa grand-mère paternelle a guidé Marina à l'extérieur du carbet. Elle se tient dos au groupe, debout sur une pierre, vêtue seulement d'un pagne noué autour de sa taille. Une lampe torche positionnée avec un sens scénographique certain permet d'observer sa silhouette immobile se détachant de l'obscurité. La grand-mère s'est assise juste derrière elle. Elle se lève tous les quarts d'heure environ pour lui verser délicatement un seau d'eau sur les épaules. Un peu avant le lever du jour, sa grand-mère maternelle prend quelques instants pour trier des éclats de pierre blanche préparés à l'avance, en testant leur tranchant sur la paume de sa main. L'autre grand-mère la rejoint. Une première ligne est tracée sur le dos de Marina. Le sang perle légèrement. Seul un frisson réprimé donne une idée de son ressenti. Les deux vieilles femmes tracent avec précision les lignes obliques formant le motif « arêtes de poisson » sur toute la surface du dos, puis des bras, de la poitrine et des jambes de la jeune fille. Son visage est crispé, ses yeux fermés, elle s'appuie fermement sur un bâton de bois d'arc (Brosimum guianense). Toutes les personnes présentes se sont levées et forment un demi-cercle autour d'elle. Deux femmes utilisent la lampe torche de leur téléphone pour mieux éclairer la scène. Les conversations sont animées, des rires les ponctuent.

\footnotetext{
${ }^{2}$ Tous les prénoms ont été modifiés.

${ }^{3}$ Note de l'éditeur: Dans cet article, la description du rituel sera présentée dans cette disposition différente.

${ }^{4}$ Terme utilisé en Guyane pour désigner les constructions ouvertes, d'origine tupi.
} 
Une fois les scarifications achevées, l'une des grands-mères prépare un seau d'eau dans lequel elle écrase des piments. Elle verse ce contenu sur une Marina stoïque à l'aide d'une calebasse. L'odeur du piment pique le nez des personnes présentes dans l'assemblée. Un trou a été creusé la veille à l'emplacement prévu afin de recueillir le jus pimenté. La jeune fille est ensuite rincée à grande eau, tandis que sa mère lui frotte le corps à l'aide de feuilles de coton.

Un soleil embrumé émerge autour de six heures du matin. Pendant que Marina est couverte de roucou (Bixa orellana) par ses grand-mères et invitée à servir de la bière de manioc aux personnes présentes, son grand frère s'enfonce dans la forêt, à la recherche d'une fourmilière repérée quelques jours plus tôt. À son retour, des fourmis noires (Paraponera clavata) de la taille d'une phalange s'agitent dans la bouteille en plastique qu'il tient en main. Des oncles et grands-pères ont déjà commencé à tresser des folioles de palme. Les fourmis sont ensuite insérées dans ces vanneries à l'aide d'une hampe de plume. Coincées entre le thorax et l'abdomen, elles se débattent en émettant un crissement sourd, et les hommes approchent le piège de leur oreille pour estimer leur état d'excitation.

Marina est amenée par sa mère devant son grand-père maternel. Celui-ci lui applique le piège à fourmis, dards en avant, sur le front, puis sur les chevilles. Sa mère la tient serrée contre elle, comme pour la protéger tout en l'empêchant de se dérober. Vient le tour de la grand-mère maternelle, qui pose les fourmis sur les poignets, les paumes et le ventre. Cette fois, Marina ne peut réprimer les frissons d'appréhension, de douleur et de fatigue qui agitent ses bras et ses jambes. Sa mère l'emmène enfin dans la maison, où elle a le droit de se reposer.

Après elle, toutes les personnes présentes profitent avec enthousiasme de l'occasion de se faire piquer par les anciens, ou de faire piquer les jeunes enfants (nettement moins enthousiastes). Les grands-mères proposent aussi aux jeunes hommes de réaliser des quadrillages avec les éclats de pierre blanche sur leurs bras et leur torse, puis de leur appliquer du jus de piment sur les scarifications. La bière coule à flot tout au long de la journée, du gibier accompagné de galette de manioc ou cassave est servi aux invités, et dans l'après-midi ceux-ci se mettent à danser au son des musiques brésiliennes et caribéennes émises par les haut-parleurs branchés à un groupe électrogène.

Avant la nuit, Marina se fait couper les cheveux par son grand-frère. 
Pour pouvoir qualifier un peu plus précisément ce rite et essayer d'en comprendre la logique, il faut prendre en compte les prescriptions qui l'entourent. Les femmes qui nous ont raconté ce moment de leur vie nous ont fait part d'une multitude d'interdits et de dangers. La jeune fille en réclusion doit par exemple faire très attention à ne pas toucher le sol de ses pieds nus, sous peine que des entités malveillantes (mabae ay, « des mauvaises choses ») entrent dans son corps et lui causent par la suite des douleurs articulaires. Elle ne peut pas non plus aller se baigner au fleuve, car son odeur attirerait le dangereux anaconda (Eunectes murinus), qui tenterait de la séduire et de l'emmener sous l'eau. L'abattis est également dangereux pour elle, le maître du manioc en particulier pouvant chercher à s'introduire dans son corps.

Figure 1. Marina est rincée avec de l'eau après le bain de jus de piment (Photo A. Schmeltz, 2018)

Grenand (1984: 18) indique en s'appuyant sur l'étude des dispositions prises à la naissance des enfants que « les interdits sont chez les Wayãpi une pratique sociale courante, 
associée à chaque moment de la vie ou la jonction entre le monde des hommes et celui des esprits forestiers est en tension ». C'est cette tension qui apparaît clairement à travers la menace de la rencontre avec l'anaconda et les attaques d'esprits. On peut également concevoir cette période comme « une nouvelle naissance assortie d'une nouvelle fragilité face aux esprits maléfiques », ainsi que le font les Shipibo-Conibo (Morin et d'Anglure, 2007: 190).

En effet, entre ses premières règles et le moment où ses cheveux ont repoussé jusqu'à ses épaules, une jeune fille voit son alimentation surveillée et nombre d'interdits alimentaires lui sont imposés sous peine de dérèglements corporels. Par exemple, manger du tapir (Tapirus terrestris) l'exposerait à avoir un gros ventre pour le reste de sa vie ; le pécari ( Tayassu pecari) lui donnerait un air méchant et des cheveux blancs; l'ara (Ara macao) la ferait saigner abondamment ; le daguet rouge (Mazama americana) la rendrait folle... Clara m'a fait part des rencontres en rêve auxquelles s'exposent les jeunes filles trop gourmandes:

Ma mère m'a un peu expliqué, pourquoi on ne doit pas manger, pourquoi on doit faire attention à tout ce qu'on mange. Parce que si moi je mange le kwata [singe atèle], après si je dors dans mon rêve y a une sa'i [grandmère] qui va venir me parler. Si tu manges le kwata [Ateles paniscus] maintenant, t'aurade trucs dans tes cheveux. Ça gratte, ça gratte, tes cheveux vont tomber. C'est pour ça qu'on fait attention, après les deuxièmes règles.

$\mathrm{O}$ - Ça, c'est si tu manges du kwata. Est-ce qu'on t'a dit une autre histoire pour un autre animal?

C - Oui, les autres animaux... Si tu manges le kaliaku [daguet gris Mazama gouazoubira] [...] et après si tu dors, dans tes rêves c'est pareil, y a une grand-mère qui va venir te parler. [...]

Voici une hypothèse sur le mode d'action des animaux ou de leurs maîtres : en transmettant leurs caractéristiques aux jeunes filles, les animaux chercheraient à transformer celles-ci en un représentant de leur propre espèce. Cette métamorphose est racontée de façon très explicite dans une histoire que nous avons recueillie, qui conte comment une jeune fille est transformée en pécari : emmenée en forêt par son mari alors qu'elle aurait dû rester en réclusion au moment de ses premières règles, elle est séduite puis emportée par un troupeau de pécaris. Elle finit par se transformer à son tour en pécari, et quand son mari la retrouve et la ramène au village elle se laisse dépérir jusqu'à mourir.

Vilaça (2002: 349), en s'appuyant sur les travaux de Lima (1995) et de Viveiros de Castro (1986) évoque ce péril transformiste à l'échelle de l'Amazonie : «Toute l'enfance, et même plusieurs périodes de la vie adulte (en particulier l'initiation, la première menstruation, la réclusion guerrière, et la maladie) sont particulièrement marquées comme hautement sensibles, impliquant très souvent la possibilité de la perte d'une identité proprement humaine. » Il s'agit donc d'une période pendant laquelle le corps de la jeune fille est poreux et malléable, ce qui justifie les interdits alimentaires et comportementaux mais 
aussi les traitements appliqués au cours du rituel de la descente du hamac. En effet, comme pour la consommation des aliments, les effets attendus des traitements physiques se feront sentir au cours de toute la suite de l'existence de la femme, s'ils sont appliqués lors de cette disposition physique particulière que sont les premières règles.

Le rapprochement de ce moment avec une nouvelle naissance est également opéré chez les Airo Pai ${ }^{5}$, à propos desquels Belaunde (2016:21) dit qu'ils considèrent que « tout le corps [de la jeune fille lors de sa première menstruation] est tendre comme celui d'un nouveau-né, et peut donc rester marqué par tous types de traits négatifs, tant moraux que physiques» C'est pourquoi on attend des scarifications qu'elles rendent la jeune fille obéissante, du coup de bâton ou de pédicelle de palmier administré par une grand-mère qu'il lui donne une démarche rapide, ou encore des piqûres de fourmis qu'elles transmettent directement la force et la motivation des personnes qui appliquent le piège à insectes (choisies parfois en-dehors de la parentèle proche en vertu de capacités de travail reconnues par la communauté) à la jeune fille. Selon l'une de nos interlocutrices, appliquer le tapiya'ilena (la bande de vannerie où sont coincées les fourmis tapiya'i - Paraponera clavata) sur le front d'une jeune fille a pour but d'« enlever la méchanceté ». Celle-ci fait l'objet du même antidote que pour lutter contre la paresse. Erikson (1996: 280-286) a mis en avant une certaine équivalence entre ces deux défauts de caractère pour les Matis, la mesquinerie étant associée dans leur éthique au refus d'accomplir des efforts visant à assurer une production pouvant être partagée avec le groupe. Heckler (2004: 250) pointe également une « obsession de la paresse » chez les Piaroa, tout comme Descola (1986: 293) chez les Achuar.

Un autre effet des traitements apparaissant en filigrane du témoignage de plusieurs femmes est l'entraînement à la résistance. Celle-ci se traduit par l'immobilité attendue des jeunes filles sous les seaux d'eau froide, au cours des scarifications puis des piqûres de fourmis, ou encore sous le coup de bâton. Plusieurs femmes mettent en avant la nécessité de ne pas pleurer et de maîtriser ses tremblements, car cette capacité pourra être nécessaire à d'autres activités ou situations. Aurélie m'explique ainsi les effets des bains d'eau froide:

Quand ils vont te laver avec de l'eau, quand après, tu trembles après, ben après... Plus tard, quand tu pourras porter du katoury [hotte agricole de portage], c'est ça, ça qui va venir sur toi, que tu vas trembler, c'est pareil, c'est presque pareil.

Le moment de la descente du hamac correspond ainsi à un travail effectué par la communauté sur le corps des jeunes filles, lesquelles participent, par leur résistance affichée, à fixer dans ce corps les capacités qui feront d'elles des travailleuses motivées et des épouses stoïques. C'est la somme de ces prescriptions et traitements qui, en conditionnant son devenir, permet de brosser le tableau de la femme wayãpi idéale : énergique, efficace, disciplinée, au physique jeune et avenant. Au moment de ses premières règles, elle se trouve au centre d'un réseau d'intentions antinomiques, au carrefour des influences de plusieurs

\footnotetext{
${ }^{5}$ Peuple de langue tukano occidental vivant en Amazonie péruvienne, colombienne et équatorienne.
} 
acteurs humains et non-humains. La rigueur avec laquelle elle observe les différents interdits vise à maintenir son propre corps protégé des entités malveillantes; à l'inverse, les traitements prodigués lors de sa descente du hamac sont appliqués au cours de ce moment de sensibilité particulière pour que leurs effets imprègnent la jeune fille le plus efficacement possible.

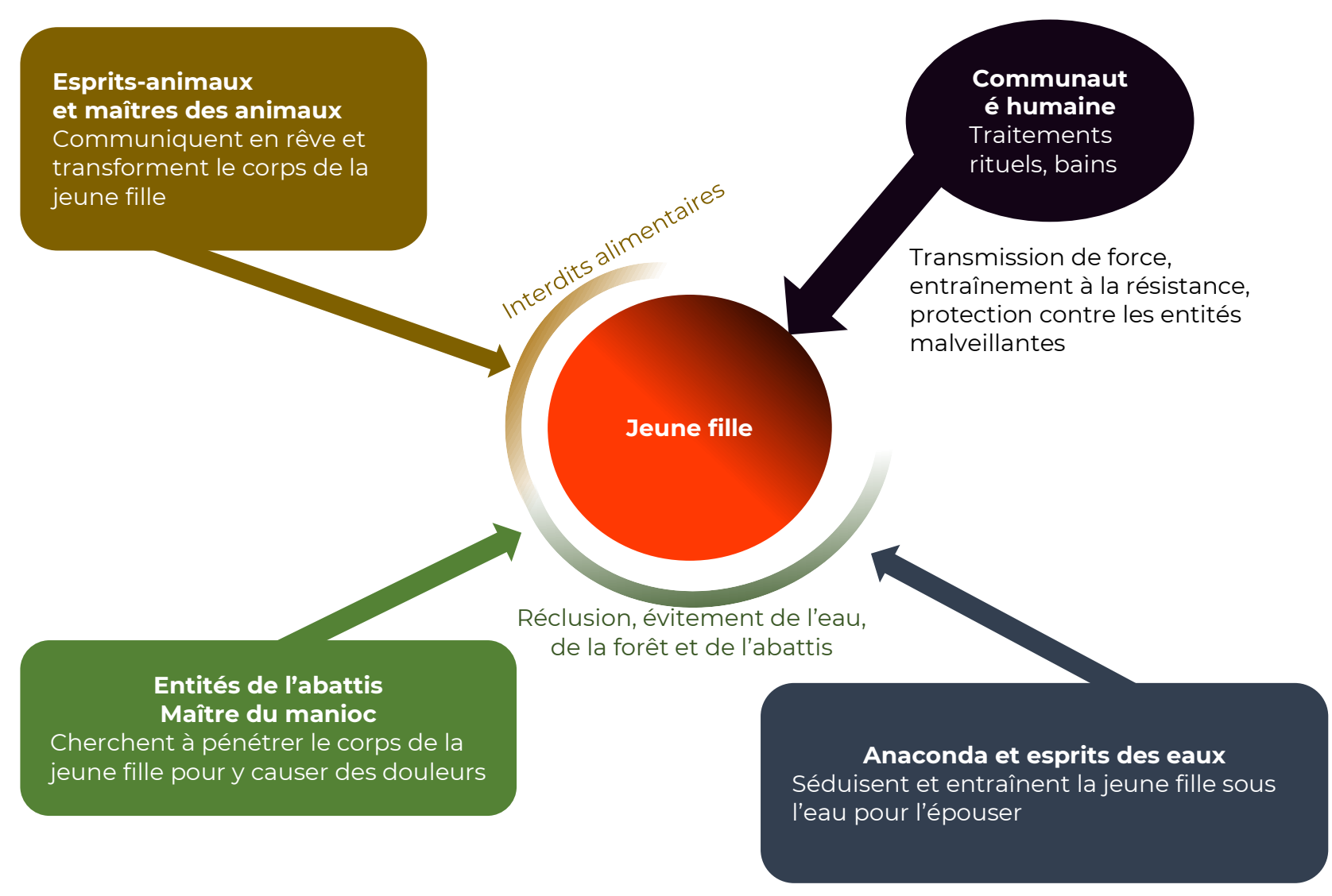

Figure 2. Les différences forces agissantes sur le corps de la jeune fille (réalisation O. Braconnier).

Le côté spectaculaire de cette cérémonie fait partie de ses caractéristiques reconnues et attendues par mes interlocuteurs ; notre présence à la descente du hamac de Marina découle en effet principalement de la demande qui nous a été faite par son grand frère de filmer toute la séquence, afin d'en conserver une trace « pour que les membres de la famille absents puissent voir ». Cet aspect n'est cependant pas forcément prioritaire pour assurer l'efficacité des gestes rituels : une amie m'a en effet raconté qu'au cours de la descente du hamac de sa belle-fille, elle a profité de la capture des fourmis pour piquer sa nièce, qui n'avait pas bénéficié de ce traitement lors du rituel organisé pour elle.

Il est cependant malaisé de parler ici d'un rituel d'initiation, dans la mesure où le secret est absent du dispositif, et où l'entrée dans un cercle spécifique d'initiées ne nous a jamais été racontée comme telle par les femmes avec qui nous nous sommes entretenues. Les réponses à notre question systématique « y a-t-il des choses que tu n'as commencé à faire qu'après la réclusion des premières règles et la descente du hamac ? » sont négatives ; les 
femmes avaient déjà commencé à apprendre les gestes de la transformation du manioc avant ce moment. Seule la possibilité de boire de la bière de manioc leur est ouverte, mais il s'agit là d'une observation personnelle et non d'un fait d'importance pour elles. En revanche, la tristesse de quitter le monde de l'enfance a parfois été évoquée. L'expression « passer le maraké »a été employée à plusieurs reprises, montrant que mes interlocutrices francophones y voient bien une épreuve et une transition : on pourra donc parler de rite de passage à l'âge adulte.

\section{Des femmes qui font des femmes}

Les caractéristiques principales du rituel étant établies, nous voulons maintenant questionner le rôle des femmes dans la mise en place du dispositif et, réciproquement le rôle du rituel dans l'expérience de ces femmes.

On a vu avec l'exemple de la descente du hamac de Marina que les femmes de sa parentèle prennent en charge la majorité des évènements. D'après les témoignages récoltés, ce sont le plus souvent les grands-mères paternelle et maternelle qui décident du moment de la descente et de la séquence des gestes. Ce sont toujours elles qui administrent le coup de bâton destiné à « faire marcher vite » et qui réalisent les scarifications. Le cas de la piqûre par les fourmis est mieux partagé (nous y reviendrons).

Une continuité entre les générations de femmes est instaurée lors de ce rituel. Clara, à propos de l'organisation future de la descente du hamac de ses filles, précise:

Si ma mère est toujours là avec moi, je vais dire à ma mère de faire comme grand-mère a fait pour elle [c'est-à-dire une version différente de ce qu'elle-même a vécu].

Elle trouve là une occasion de lier les capacités physiques et donc le corps de sa fille avec celui de sa grand-mère, à travers les divers gestes que l'une réalisera sur l'autre. Mais l'intégrité de la chaîne générationnelle est indispensable dans certaines familles: une autre amie m'a en effet expliqué que sa mère étant décédée lorsqu'elle était jeune, elle n'avait pas pu être piquée par les fourmis et recevoir ce type de transmission.

À propos du rôle du rituel dans la vie des participantes adultes présentes, il est intéressant de mentionner « celle qui a fait descendre ». C'est une relation de parenté (la mère, la tante, ou parfois la grande sœur de la jeune fille) qui entraine l'accession à ce statut, synonyme de responsabilisation puisque l'avenir de la jeune fille descendue est placé entre les mains de celle qui organise le rituel. Un ensemble de savoirs sur les modalités de relations avec les êtres environnants (animaux, maîtres des animaux, esprits de la forêt et esprits des morts) est nécessaire pour mener à bien non seulement la descente du hamac mais aussi toute la période de réclusion. Avoir « fait descendre » une fille génère ainsi une certaine forme de reconnaissance de la part de la communauté. Mais celle-ci est aussi étroitement liée au fait d'avoir organisé une fête de bière et donc d'avoir produit celle-ci. Audrey souligne l'importance de ce moment pour la mère de la jeune fille: 
Dès que ma fille avait ses premières règles, c'est là que je me suis dit. Ça a changé, j'ai une grande responsabilité de ma famille.

L'étape du processus qui suit les deuxièmes règles doit être citée ici : il s'agit du moment où l'on demande aux jeunes filles de procéder seules à la fabrication de la bière de manioc. Au cours de trois jours de travail intense, elles sont mises à l'épreuve. L'objectif est double: il s'agit de tester la solidité de leurs savoir-faire en matière de transformation du manioc, indispensables à la vie de femme ${ }^{6}$, mais aussi de profiter de la malléabilité de leur corps pour lui donner une efficacité à long terme. Clara nous a rapporté ce que sa grandmère disait à sa mère lorsque celle-ci était fatiguée de râper le manioc:

Après sa deuxième règle, [la] mère [de ma mère] est partie chercher le manioc à l'abattis, au moins deux, trois, quatre terrines [marmites de 200 litres], y a que ma mère, mmmh ! Et puis ma mère m'a dit, je ne peux pas tout finir. Et ma grand-mère a dit à ma mère : «Tu finis, si tu ne finis pas tu vas être comme un tortue. Si tu finis, là tu vas être comme un écureuil» ${ }^{7}$

Les jeunes filles construisent elles-mêmes leur corps de femme au cours de ce travail : on les prévient qu'elles doivent accomplir les gestes le plus rapidement possible pour rester efficaces tout au long de leur vie. On retrouve le même genre de considérations ailleurs en Amazonie, par exemple chez Airo Pai, où c'est une grande quantité de maïs que les jeunes filles doivent moudre «pour que leurs bras s'endurcissent» (Belaunde, 2001: 126). L'apprentissage des gestes de la transformation du manioc est inséparable de celui des savoirs liés à la matière. Comme chez les Kaxinawá, on devine chez les Wayãpi l'idée que «la production corporelle doit être parallèle à la production de la mémoire et du savoir chez la personne» (McCallum, 1999: 5). Cette production conjointe, si elle connaît une intensité remarquable et publique au moment des premières règles, a cependant commencé plus tôt dans la vie des jeunes filles, qui observent et imitent leur mère dès qu'elles en ont la possibilité. La construction du corps se poursuit aussi après les premières règles, comme l'atteste le fait que les personnes convoquées pour piquer les jeunes filles avec les fourmis, et ainsi leur transmettre force et savoir, sont toujours des personnes âgées, parvenues au plus haut degré de maîtrise de leurs arts. Comme le note Kensinger (1992: 246), en Amazonie, «le corps est un corps qui sait et qui est continuellement en processus de fabrication, par l'incorporation de pensées».

On observe avec l'expression de sa puissance dans le cadre d'une activité exclusivement féminine que le corps de la jeune fille devient le lieu d'une «performance » de genre (Butler, 2005 [1990]: 266) ; à partir de cette première transformation du manioc et

\footnotetext{
${ }^{6}$ Rappelons ici que pour les Wayãpi la culture du manioc amer et doux est la base de leur alimentation. Il ne se passe pas une journée dans leur vie sans quils consomment du manioc sous forme de galette, d'empois ou de bière.

${ }^{7}$ La tortue terrestre (yãwi) étant dans la culture wayãpi l'archétype de la lenteur tandis que l'écureuil (kusipuru) est vu comme un animal très actif et industrieux.
} 
au cours de toutes les suivantes, c'est la preuve d'une force et d'une résistance de femme idéale qu'il doit donner à voir. Nous rejoignons ici Guzmán (1997: 55, citée par Belaunde, 2005: 44), pour qui «le genre d'une personne, dans le cas des Canelos, se développe et se complète dans la mesure où celle-ci acquière certaines habilités, les mêmes qui s'incorporent au corps d'une personne et s'extériorisent dans certains produits».

On a dit plus haut que des hommes étaient présents à toutes les étapes du rituel. Contrairement à ce qui se passe dans certains rites initiatiques masculins, notamment dans l'ensemble Tukano (Hugh-Jones, 2011 [1988]: 158) la descente du hamac ne contient pas de message sous-jacent destiné à l'autre genre, avec lequel l'identité des protagonistes de la cérémonie devrait se construire en opposition. L'effet le plus évident de ce rituel, sa réalisation immédiate, est de souder les membres de la communauté, femmes et hommes et jeunes, autour de la consommation collective de bière de manioc, comme toujours dans les évènements sociaux wayãpi. Les hommes sont en effet non seulement présents au cours de toute la cérémonie mais nécessaires à certaines phases : on attend de ceux de la parentèle qu'ils ramènent des produits de la chasse et de la pêche pour sustenter les invités, ainsi que les fourmis tapiya'i, dont ils ne peuvent connaître la localisation que grâce à leur fréquentation assidue de la forêt. Le geste de la transmission de force via les fourmis est mixte : nos interlocutrices nous ont expliqué que les jeunes filles peuvent être piquées par des hommes, des femmes, ou bien par les deux, à condition que leur capacité de travail soit reconnue par la communauté. Certaines femmes nous ont indiqué que certains endroits du corps des jeunes filles sont piqués avec un objectif précis, par des personnes d'un genre ou de l'autre : les hommes appliquent le piège à fourmis sur le front des jeunes filles, afin de leur donner une détermination sans failles, tandis que les vieilles femmes leur piquent le ventre pour qu'elles aient de la force au moment d'accoucher. On peut ainsi retenir que le rituel de la descente du hamac, bien qu'organisé par des femmes pour une femme, ne constitue pas un moment d'exclusion des hommes. L'idée n'est pas ici d'inclure ou d'exclure, mais de rassembler. Si une séparation est opérée par le rituel, c'est celle qui distingue et distancie les collectifs de personnes humaines et ceux des animaux et des esprits dont on veut protéger les jeunes adultes.

\section{L'autre descente du hamac}

Certaines femmes interrogées ont parlé d'un pendant masculin du rite de passage féminin, qui ne serait plus que rarement accompli, au moment où la voix des jeunes hommes mue. Quasiment toutes nos interlocutrices établissent cependant spontanément le lien entre les interdits et les rites des premières règles et ceux qui entourent chez un homme le moment de la naissance de son premier enfant. Voici la façon dont Clara établit ce lien, alors qu'elle nous explique les effets de la transgression d'un interdit alimentaire par une jeune fille en réclusion

Une biche, par exemple si tu la manges, et après si tu dors, dans tes rêves y a une grand-mère qui va venir te parler : "Voilà, tu la manges, tu ne respectes même pas !" Tes cheveux vont devenir blancs. Regarde Gabin, c'est pareil. C'est comme ça, c'est pareil avec Gabin, c'est un garçon mais 
avec quand il a eu son premier fils il a mangé de la biche et du coup il a les cheveux blancs.

Bien qu'elles constituent un tout cohérent, il faut ici établir une distinction entre les règles qui visent à protéger le nouveau-né de menaces extérieures, et celles dont la transgression aboutit à l'altération de l'état du père. Or, ces dernières sont d'après mes interlocutrices les mêmes que pour la jeune fille au moment de ses premières menstruations. Comme elle, juste après la naissance de son premier enfant, le jeune père s'isole une semaine dans un hamac, ne mange que des petits poissons et de la galette de manioc pendant plusieurs mois. Il évite certains gibiers pour ne pas devenir gros, avoir des cheveux blancs ou encore abîmer ses dents. Le même risque de transformation le guette en cas de transgression ; un mythe recueilli chez les Wayãpi de Trois-Sauts ${ }^{8}$ raconte par exemple qu'un père qui n'avait pas respecté certains interdits alimentaires à la naissance de son enfant se transforma en singe atèle (Grenand, 1982: 273). Et comme pour la jeune fille, les femmes de la maisonnée préparent de la bière en vue de sa descente du hamac, au cours de laquelle il est piqué par des fourmis, scarifié et peint au roucou.

Cette similarité entre les deux rituels indique que ces deux stades de vie, puberté pour les unes et paternité pour les autres, sont placés sur un plan d'équivalence en ce qui concerne la sensibilité des corps. Les chroniqueurs du XVIème siècle comme Thevet (1557, cité par Métraux, 2014 [1928]: 213) montraient déjà la proximité entre ces deux types de réclusion, et l'état du corps qui les justifiaient. Comme lors des rites funéraires, et ceux entourant un guerrier après un homicide, il s'agissait d'encadrer des moments où les personnes traversaient « une dangereuse période de transformation, au terme de laquelle elles émergeaient rénovées, comme si elles venaient de renaître »(Belaunde, $2016: 15$ ).

Le parallèle entre les descentes du hamac masculine et féminine invite à relativiser l'a priori essentialiste qu'aurait pu inspirer la liste des prescriptions faites aux jeunes filles. La préservation d'un corps dans un état d'ouverture périlleux et la transmission d'une force permettant à l'individu de s'inscrire dans la communauté en tant que membre productif sont en effet chez les Wayãpi au cœur des préoccupations, quel que soit le genre. Les femmes et les hommes, bien qu'amenés à réaliser des tâches différentes et complémentaires au cours de leur vie d'adultes, ne sont donc opposés ni au cours du rituel ni après. Or, au lieu d'évoquer un caractère spécifiquement féminin ou masculin des différents traitements, mes interlocutrices ont mis en avant l'équivalence pratique et logique de ces traitements dans le parcours des jeunes adultes. Contrairement à ce qu'observe Strathern en Mélanésie (1988 : 107) l'attention accordée au pouvoir reproducteur des femmes est tout au long de la séquence rituelle assez secondaire : seule une piqûre de fourmi sur le ventre appliquée par une de ses grands-mères a pour objectif de préparer la jeune fille à ses futurs accouchements. Tous les autres traitements visent à la rendre rapide, résistante, forte et courageuse.

\footnotetext{
${ }^{8}$ Les Wayãpi sont répartis en trois communautés distinctes bien que parentes. En Guyane française, ils vivent dans la commune de Camopi, en deux communautés séparées : les Wayãpi de Trois Sauts en amont du fleuve Oyapock et les Wayãpi de Camopi sur le Moyen-Oyapock, là où nous avons mené notre étude. La troisième communauté, plus isolée des deux premières, se situe dans la zone de l'Amaparí dans l'État brésilien de l'Amapá.
} 
Pour revenir aux jeunes pères, leur moment de réclusion dans le hamac fait suite à une sorte de seconde naissance, et les mots d'Alès (1998: 27) à propos des Yanomami me semblent particulièrement appropriés pour décrire cette transition : « Les enfants font "naître" le père, engendrent son développement physique et achèvent sa maturation physique. Pour ainsi dire, c'est l'enfant qui permet la création du père, puisque l'homme doit reproduire pour être produit, puisqu'il doit créer pour exister lui-même. Constatons que ce faisant il tend à s'auto-reproduire» Rival (1998: 634) parle également de renaissance, en incluant la mère dans son analyse, et elle considère que la diète particulière adoptée par les parents prouve cet état de fait, puisqu'en faisant « suivre aux parents d'un nouveau-né le régime alimentaire d'un nourrisson » ceux-ci revivent « pendant toute la période de jeûne les premières années d'un enfant ». Ainsi, le terme kwaku désigne en wayãpi la posture immobile de la personne en réclusion dans son hamac, en référence à la position de la poule protégeant ses petits en devenir. Dans le cas d'une naissance, le fait de ne pas quitter le nid est nécessaire pour garantir la protection de l'enfant comme celle des parents. Mais comment interpréter l'utilisation du terme kwaku à propos d'une jeune fille recluse au moment de ses premières règles ? Suivant les arguments de Rival (ibid.) et Alès (ibid.), il faut peut-être comprendre, que c'est à elle-même qu'elle donne naissance.

Afin de nuancer légèrement la vision égalitaire dépeinte à propos de la descente du hamac, il convient de rappeler que la complémentarité entre femmes et hommes - qui est au cœur de l'organisation sociale des Wayãpi - implique aussi que le rituel de la descente du hamac est là pour préparer les premières à leur futur rôle d'épouses dévouées. Sofia m’a par exemple évoqué en ces termes les suites de ses premières règles:

Le souvenir que j'ai gardé c'est quand je faisais des choses toutes seules, je faisais la galette de manioc toute seule, le cachiri toute seule, tout ce qui est partie cuisine toute seule, et là mes grands-parents m'ont dit : "Voilà, t'es devenue une femme maintenant". Ils m'ont dit : "T'es devenue une femme et t'as le droit d'avoir un mari un jour". Si j'étais incapable de faire tout ça et bien ils vont me dire : "Mais t'es incapable, tu ne mérites pas avoir un mari, parce que tu ne pourras pas le nourrir, prendre soin de lui", c'est ce qu'on me disait à moi.

On note dans cette citation une sorte de pression subie par la jeune fille, pour qui « avoir un mari un jour » représenterait le couronnement d'efforts tendus vers l'acquisition de capacités lui permettant d'assurer l'autonomie alimentaire de son foyer. ${ }^{9}$

\section{Conclusion}

\footnotetext{
${ }^{9}$ Dans la société wayãpi, cette autonomie alimentaire n'est possible que si le futur époux tresse les vanneriesoutils pour sa femme. En effet, traditionnellement, un jeune homme se doit de maîtriser la confection de nombreuses vanneries indispensables à la transformation du manioc en aliment (Davy, 2007). De même, afin d'être considéré comme un homme, un wayãpi doit savoir chasser, pêcher et travailler le bois.
} 
Il est important de préciser pour conclure que les femmes wayãpi ne considèrent pas que la période des menstruations implique un état de saleté, polluant et néfaste pour la communauté. Il s'agit avant tout d'une période d'ouverture particulière, au cours de laquelle puissance potentielle et fragilité face aux non-humains caractérisent en même temps le corps de la jeune femme en construction. Buckley et Gottlieb (1988: 51, repris par Belaunde, 2001: 248) soulignent ainsi avec justesse que « le sens de la menstruation dans chaque culture doit être compris dans son propre contexte, et qu'on ne peut lui attribuer une valeur a priori. Les idées et pratiques menstruelles [...] manifestent des concepts originaux sur la créativité, la santé, et les relations de genre et de pouvoir spécifiques à chaque société. » Ces idées et leur manifestation sous une forme ritualisée nous paraissent en tous cas se présenter avec une grande constance au fil des générations successives. Jusqu'à aujourd'hui, il semble que les regards extérieurs - qu'ils soient brésiliens, français ou créoles - n'ont pas affecté la façon dont les mères perçoivent la renaissance de leurs filles lors de leurs premières règles, et les précautions qu'elles considèrent nécessaires de prendre à ce moment. Cette permanence du rituel nous paraît fortement liée au fait qu'une femme wayãpi ne peut devenir adulte sans un corps capable d'assumer toutes les tâches liées à l'agriculture, à la transformation du manioc et au brassage de la bière, tandis que la proximité avec les entités de la forêt constitue la même menace de nos jours que du temps des grands-mères de nos interlocutrices. La préparation et la protection du corps des femmes en devenir constituent dès lors un ensemble de gestes plus pragmatiques que symboliques, ce qui explique pourquoi le déroulement du rituel ressemble si fortement à ce que décrit le chroniqueur Thevet au XVIème siècle chez les Tupinamba. On peut aussi voir dans cette constance des idées et des gestes une des manifestations de la résistance des femmes face aux processus plus ou moins volontaires d'acculturation engendrés par la présence de l'État français - scolarisation obligatoire, sédentarisation, versement des aides sociales - et par la proximité avec les commerçants brésiliens situés juste en face sur la rive brésilienne de l'Oyapock, qui mettent à disposition toutes sortes de denrées alimentaires et de boissons alcoolisées. ${ }^{10}$

Si la notion de genre peut sembler à première vue importante dans la mise en place $\mathrm{du}$ dispositif rituel qui encadre les premières règles, l'observation de la descente du hamac masculine invite à reconsidérer avec prudence les catégories réellement opérantes dans les attendus du groupe vis-à-vis des individus adultes, qui ne diffèrent finalement pas fondamentalement en fonction du genre des personnes, puisque la motivation au travail et la résistance à l'effort constituent les qualités primordiales que le groupe cherche à transmettre aux corps en construction.

On peut donc questionner l'existence d'une forme de reproduction parallèle dans le rituel de la descente du hamac, ce moment où des femmes fabriquent d'autres femmes. Nous pensons que cette forme de transmission n'est pas exclusive d'autres situations dans lesquelles la frontière entre les genres se fait plus poreuse. Nous rejoignons ici Belaunde,

\footnotetext{
${ }^{10}$ Ce processus de résistance et d'adaptation de la société wayãpi de Camopi a également été mis en évidence dans le cadre de leur réappropriation territoriale : Davy et al. (2012) ont montré l'échec de la sédentarisation imposé par l'Etat français en expliquant comment depuis une vingtaine d'années les Wayãpi ont créé des dizaines de nouveaux villages, se réappropriant ainsi leur zone historique de peuplement.
} 
lorsqu'elle écrit que : « les deux conceptions du genre, croisées et parallèles, coexistent dans le tissu social amazonien ; selon le contexte et le moment du cycle de vie des personnes, l'une devient une toile de fond, tandis que l'autre se place au-devant de la scène de manière dynamique. Il est donc possible de faire une lecture des relations entre hommes et femmes qui présente des aspects des deux conceptions entremêlées » (Belaunde, $2005: 24$ ). Lors de la descente du hamac d'une jeune fille, la reproduction parallèle occupe le devant de la scène. Les éléments rituels et symboliques qui interviennent à cette occasion s'assemblent selon une logique qui lie ensemble les âges de la vie : ce qui se joue au moment des premières règles conditionne les capacités des futures mères et cultivatrices, tandis que les mères et les grandsmères sont indispensables à la consolidation et à la protection du corps de leurs filles et petites-filles.

\section{Bibliographie}

ALES, Catherine. 1998. «Pourquoi les Yanowamï ont-ils des filles? ». In : GODELIER, Maurice; PANOFF, Michel (dir.). La Production du Corps. Paris : Editions des Archives Contemporaines, Overseas Publishers Association, pp.281-315.

BELAUNDE, Luisa Elvira. 2001. Viviendo bien: género y fertilidad entre los Airo-Pai de la Amazonía Peruana. Lima: Centro Amazónico de Antropología y Aplicación Práctica.

BELAUNDE, Luisa Elvira. 2005. El recuerdo de luna: género, sangre y memoria entre los pueblos amazónicos. Lima : Centro Amazónico de Antropología y Aplicación Práctica.

BELAUNDE, Luisa Elvira. 2016. Woxrexcüchiga: el ritual de la pubertad en el pueblo Ticuna. Perú, Ministerio de Cultura.

BELLIER, Irène. 1991. « Le genre, la nature et les hommes chez les Mai Huna (Amazonie péruvienne) ». Journal des anthropologues, n. $45: 29-38$.

BRACONNIER, Olivia. 2021.

BUCKLEY, Thomas; GOTTLIEB, Alma. 1988. Blood magic. Berkeley, CA: University of California Press.

BUTLER, Judith. 2005 [1990]. Trouble dans le genre. Pour un féminisme de la subversion. Paris: La Découverte.

DAVY, Damien. 2007. «Vannerie et vanniers": approche ethnologique d'une activité artisanale en Guyane française ». Thèse de doctorate en anthropologie.Université d'Orléans.

DAVY, Damien; TRITSCH Isabelle; GRENAND, Pierre. 2012. «Construction et restructuration territoriale chez les Wayãpi et Teko de la commune de Camopi, Guyane française ». Confins, n. 162.

DESCOLA, Philippe. 1986. La Nature domestique: symbolisme et praxis dans l'écologie des Achuar. Les Editions de la MSH.

ERIKSON, Philippe. 1996. La griffe des aïeux: marquage du corps et démarquages ethniques chez les Matis d'Amazonie. Leuvan: Peeters Publishers. 
GRENAND, Françoise. 1984. «La longue attente ou la naissance à la vie dans une société Tupi (Wayampi du Haut Oyapock, Guyane Française) ». Bulletin de la Société Suisse des Américanistes, , vol. 48 : 13-27.

GRENAND, Françoise. 1982. Et l'homme devint jaguar: l'univers imaginaire et quotidien des Indiens Wayãpi de Guyane. Paris: L'Harmattan.

GUZMÁN, María Antonieta. 1997. Para que la yuca beba nuestra sangre. Trabajo, género y parentesco en una comunidad quichua de la Amazonía ecuatoriana. Quito: Abya-Yala.

HECKLER, Serena. 2004. « Tedium and creativity: the valorization of manioc cultivation and Piaroa women ». Journal of the Royal Anthropological Institute, vol. 10, n.2: 241259.

HUGH-JONES, Stephen. 2011 [1984]. La palma y las Pléyades. Bogotá: Ediciones Universidad Central

KENSINGER, Kenneth. 1992. A body of knowledge, or, the body knows. In: Expedition, vol. 33 , n. $3: 37-45$.

LIMA, Tania. 1995. A parte do cauim: etnografia juruna. Rio de Janeiro : Ph.D. dissertation, Museu Nacional dans la Universidade Federal do Rio de Janeiro.

McCALLUM, Cecilia. 1999. «Aquisição de gênero e habilidades produtivas: o caso Kaxinawá ». Revista Estudos Feministas, vol. 7, n. 1-2 : 157-157.

METRAUX, Alfred. 2014 [1928]. La religion des Tupinamba et ses rapports avec celle des autres tribus Tupi-Guarani. Paris : Presses Universitaires de France ;

MORIN, Françoise; SALADIN D’ANGLURE, Bernard. 2007. «Excision féminine/incision masculine ou la construction sociale de la personne chez les shipibo-conibo d'Amazonie péruvienne » In : MATHIEU, Nicole-Claude (éd.), Une maison sans fille est une maison morte. La personne et le genre en sociétés matrilinéaires et/ou uxorilocales. Paris, Maison des sciences de l'homme.

RIVAL, Laura. 1998. « Androgynous parents and guest children: the Huaorani couvade ». Journal of the Royal Anthropological Institute, vol. 4. N. 4 :619-642. DOI /10.2307/3034825

ROJAS ZOLEZZI, Enrique Carlos. 1992. «Acerca del "saber cuándo hacer". El calendario Campa Asháninka y el ciclo anual de actividades ». Anthropologica, vol. 10, n. $10: 171$ 190.

SANTOS GRANERO, Fernando. 1991. The Power of Love: The Moral Use of Knowledge Amongst the Amuesha of Central Peru. London: Athlone Press.

STRATHERN, Marilyn. 1988. The Gender of the Gift. Problems with Women and Problems with Society in Melanesia. Berkeley : University of California Press.

THEVET, André. 1557. Les singularitez de la France antarctique, autrement nommée Amerique: $\&$ de plusieurs terres \& isles decouvertes de nostre temps. Paris : Clos Bruneau.

TOLA, Florencia Carmen. 2009. «Les conceptions du corps et de la personne dans un contexte amérindien. » L'Harmattan, coll. « Recherches Amériques latines », Paris, 2009. VILAÇA, Aparecida. 2002. «Making kin out of others in Amazonia ». Journal of the Royal Anthropological Institute, vol. 8, n. 2: 347-365. DOI 10.1111/1467-9655.00007

VIVEIROS DE CASTRO, Eduardo. 1986. Araweté: os deuses canibais. Rio de Janeiro: ANPOCS/Jorge Zahar Editor. 


\section{O que o ritual da descida da rede pode dizer sobre gênero entre os Wayãpi de Camopi}

resumo $\mathrm{O}$ artigo reflete sobre a experiência de mulheres Wayãpi na comuna de Camopi, na Guiana Francesa, e trata especificamente das prescrições e tratamentos comportamentais que marcam o momento da primeira menstruação. Os corpos de meninas são considerados particularmente sensíveis a ataques de entidades maliciosas. O ritual de descida da rede organizado no final do resguardo do primeiro período constitui um momento paralelo de reprodução, pois as mulheres das gerações anteriores o organizam para transmitir às meninas capacidades físicas e mentais como a motivação no trabalho. e resistência à dor. No entanto, as nossas interlocutoras Wayãpi destacam a equivalência entre o ritual da primeira menstruação para as mulheres e o do nascimento do primeiro filho para os homens, evidenciando que as principais capacidades esperadas dos adultos são as mesmas, seja qual for o seu sexo.

palavras-chaves gênero, Amazônia, ritual, puberdade, mulheres

Lo que el descenso de la hamaca dice sobre el género en tre los Wayãpi de Camopi

reumen El artículo presenta la experiencia de las mujeres Wayãpi en la comuna de Camopi, Guayana Francesa, y trata específicamente de las prescripciones y tratamientos conductuales que enmarcan el momento de su primer período. Entonces, se considera que los cuerpos de las niñas son particularmente sensibles a los ataques de entidades maliciosas. El ritual de descenso de la hamaca organizado al final del encierro del primer período constituye un momento paralelo de reproducción, pues las mujeres de generaciones anteriores lo organizan con el fin de transmitir a las jóvenes capacidades físicas y mentales como la motivación en el trabajo. y resistencia al dolor. Sin embargo, nuestras interlocutoras Wayãpi destacan la equivalencia entre el ritual de la primera menstruación para las mujeres y el del nacimiento del primer hijo para los hombres, mostrando así que las principales capacidades esperadas de los adultos son las mismas, cualquiera que sea su género.

palabras clave género, Amazonía, puberdad, mujeres

\section{autrices}

Cadernos de Campo (São Paulo, online) | vol. 30, n. 2 | p.1-18 | USP 2021

\section{Olivia Braconnier}

Après un master en botanique tropicale, Olivia Braconnier a travaillé pendant deux ans en tant que professeur des écoles à Camopi. Elle a ensuite repris un cursus en anthropologie, et sa recherche de master porte sur les processus du devenir-femme chez les Wayãpi de Camopi.

\section{Silvine Walacou}

Sylvine Walacou a grandi dans la commune de Camopi, en Guyane française, où elle a étudié jusqu'au collège, avant de poursuivre ses études dans la ville de Kourou. Elle a obtenu le baccalauréat, puis a commencé à travailler au centre de santé de Camopi comme adjointe administrative. En 2016, elle a fondé avec une médecin l'association Camopi Waiwi, dont le but est de permettre aux femmes de Camopi de se retrouver autour d'ateliers d'artisanat. En juin 2020, elle est nommée 5ème adjointe au maire de Camopi, et officie comme déléguée à la santé et à la vie associative. 
BRACONNIER; WALACOU | Ce que la descente du hamac dit du genre... $\mid 18$

Déclaration d'auteur : Les deux auteurs sont responsables de la collecte et de l'analyse des données ethnographiques. Olivia a été chargée de la rédaction de l'article

Financement: Centre National de la Recherche Scientifique

Reçu le 15 août 2021

Approuvé le 12 décembre 2021 